Published in final edited form as:

Nat Mater. ; 10(9): 704-709. doi:10.1038/nmat3095.

\title{
In vivo and in vitro tracking of erosion in biodegradable materials using non-invasive fluorescence imaging
}

\author{
Natalie Artzi $i^{1,2,{ }^{*}}$, Nuria Oliva ${ }^{1,3}$, Cristina Puron $^{1,3}$, Sagi Shitreet ${ }^{1,4}$, Shay Artzi ${ }^{5}$, Adriana bon \\ Ramos $^{1,3}$, Adam Groothuis ${ }^{6}$, Gary Sahagian ${ }^{7}$, and Elazer R. Edelman ${ }^{1,8}$ \\ ${ }^{1}$ Harvard -MIT Division of Health Sciences and Technology, Massachusetts Institute of \\ Technology, Cambridge, Massachusetts 02139 \\ 2Department of Anesthesiology, Brigham and Women's Hospital, Harvard Medical School, \\ Boston, Massachusetts 02115 \\ ${ }^{3}$ Institut Qu imic de Sarrià, Universitat Ramon Llull, Barcelona, Spain 08017 \\ ${ }^{4}$ Ort Braude College, Karmiel, Israel, 21982 \\ 5IBM Research, Hawthorne, NY 10532 \\ ${ }^{6}$ Concord Biomedical Sciences and Emerging Technologies, Lexington MA \\ ${ }^{7}$ Department ofPhysiology, Tufts University School of Medicine, Boston, Massachusetts 02111 \\ ${ }^{8}$ Cardiovascular Division, Department of Medicine, Brigham and Women's Hospital, Harvard \\ Medical School, Boston, Massachusetts 02115
}

\begin{abstract}
The design of erodible biomaterials relies on the ability to program the in vivo retention time, which necessitates real-time monitoring of erosion. However, in vivo performance cannot always be predicted by traditional determination of in vitro erosion ${ }^{1,2}$, and standard methods sacrifice samples or animals ${ }^{3}$, preventing sequential measures of the same specimen. We harnessed non-invasive fluorescence imaging to sequentially follow in vivo material-mass loss in order to model the degradation of materials hydrolytically (PEG:dextran hydrogel) and enzymatically (collagen). Hydrogel erosion rates in vivo and in vitro correlated, enabling the prediction of in vivo erosion of new material formulations from in vitro data. Collagen in vivo erosion was used to infer physiologic in vitro conditions that mimic erosive in vivo environments. This approach enables rapid in vitro screening of materials, and can be extended to simultaneously determine drug release and material erosion from a drug-eluting scaffold, or cell viability and material fate in tissue-engineering formulations.
\end{abstract}

\footnotetext{
Users may view, print, copy, download and text and data- mine the content in such documents, for the purposes of academic research, subject always to the full Conditions of use: http://www.nature.com/authors/editorial_policies/license.html\#terms

*Corresponding author: Natalie Artzi, Ph.D., Harvard-MIT division for Health Sciences and Technology, Massachusetts Institute of Technology E25-449, 77 Massachusetts Ave., Cambridge, MA 02139 (USA), nartzi@mit.edu, fax: 617-2532514.

Author contribution

N.A. conceived the ideas, designed and directed the research. N.A., N.O., C.P., S.S., A.G. and A.R. conducted the experiments. N.A. and S.A. performed the mathematical modeling. G.S. contributed reagents and technical expertise. E.R.E. participated in the data analysis and model synthesis. N.A. and E.R.E. wrote the manuscript.
} 
Biodegradable materials serve as platforms for structural stabilization, void filling, drug delivery and tissue engineering ${ }^{4-8}$ offering the promise of reduced complications posed by permanent foreign objects. ${ }^{9}$ Yet, controlled material development is limited as degradation in vivo is more complex than in vitro, and in vitro assays are rarely adequate measures of implant behavior. Loss of material integrity, structure, and eventually mass progress dependently over time but are dominated by different environmental forces in vitro and in vivo. The question remains as to whether erosion or loss of mass in one domain can predict performance in the other. Gravimetric determinations from periodic sampling of explant weight cannot follow the same formulation over time, necessitate large number of animals to follow small number of samples and have excessive variability. ${ }^{3,10,11,12,13}$. Chromatography tracks molecular weight changes ${ }^{2,12}$ but cannot be applied to eliminable materials that do not undergo chain scission. Material environment affects erosion, and the material and degradation products may affect the environment in turn ${ }^{14,15}$. Thus, in vivo residence times and in vitro durability of three dimensional degradable structures differ ${ }^{2}$.

We developed a noninvasive imaging technique that tracks material erosion in vivo through a fluorescent tag covalently attached to components of model materials. Materials erosion was calculated from the decay in total material fluorescence signal using non-invasive In Vivo Imaging System (IVIS). Model hydrolytically degradable adhesive materials used herein are based on polyethylene glycol (PEG) amine and dextran aldehyde ${ }^{16,17}$. PEG replete with amine groups and oxidized dextran react at body temperature in a Schiff base reaction to form adhesive materials as aldehydes bind to tissue amines. The reaction is reversible and the material hydrolyzes to its polymeric components ${ }^{16,17}$. Although PEG polymers may undergo enzymatic degradation, significant fluid uptake and swelling dominate the degradation of our formulated PEG:dextran hydrogels, resulting in hydrolyticsensitive materials. As material shape dictates fluid uptake, we examined whether fluorescence tracking could distinguish the fate of PEG:dextran formulations cast in a series of shapes, sizes and varied PEG solid content. Compressed denatured type II collagen was used as a model for enzymatically degradable material whose erosion profile should change with implantation site and natural variation in enzyme content.

To enable the use of tagged materials to measure erosion rate, we verified that fluorescent tagging had no effect on material properties. Indeed, swelling and erosion kinetics (Supplementary Figure 1a), gelation time (Supplementary Figure 1b) and adhesion to biological tissues (Supplementary Figure 1c) of PEG:dextran were unaffected by 5\% fluorescent PEG (demonstrated using the following formulation of dextran, D10-50-14 and PEG, P8-10-40, as described in Supplementary Table 2). Fluorescent tag tracking however provides different erosion kinetics than conventional wet gravimetric analysis. The two assays demonstrated identical duration of material integrity. Yet, while tag-tracking indicated immediate erosion upon immersion, gravimetric determinations inferred material weight gain until swelling peaked (supplementary Figure 2). Indeed, erosion determined by gravimetric analysis was $40 \%$ faster than by fluorescent tracking, as the former likely considers elution of media trapped within the network during swelling and not just erosion of polymeric chains. When the effect of swelling is eliminated and gravimetric analysis was performed after samples were dried, the erosion profile coincided with the fluorescent 
profile (supplementary Figure 2), up to the limit of scale sensitivity in the regime where fluorescent quantification remains constantly sensitive. Such drying removes samples from further experimentation and is inappropriate for in vivo implants. Fluorescent tracking allows high fidelity identification of polymeric chains released from the bulk gel sequentially from the onset of immersion and without sample destruction.

Hydrophilic material swelling and dissolution is determined by fluid uptake which is modulated by material surface area and the volume of diluent ${ }^{4}$. We examined material loss profiles in both domains for disks, blocks or hollow mesh cylinders of PEG:dextran (D10-50-14 P8-10-40, supplementary Figure 3) all containing 5\% fluorescent PEG with fixed mass. Construct erosion in vivo was calculated from efficiency signals at the region of interest around the sample (Figure 1a). Erosion was biphasic - comprised of rapid sample loss presumably through diffusion of non-crosslinked, unreacted PEG and dextran polymeric chains, followed by more gradual degradation of the crosslinked copolymer network. In each case, erosion kinetics in vivo was faster but tracked with the biphasic behavior seen in vitro (Figure 1b-c). Thin blocks and mesh cylinders with significantly higher surface area than the disks showed not unexpectedly accelerated erosion in vitro compared with the erosion in vivo, leading to exponential dependence of the ratio between in vivo and in vitro erosion with time (Figure 1d, $\mathrm{R}=0.99$ for both shapes). In contrast, disk erosion was diluent volume limited and in fixed volume environments in vivo erosion kinetics tracked linearly with erosion in vitro (Figure 1d, $\mathrm{R}=0.98$ ).

Within each hydrogel shape, formulation protocols control erosion profiles as network formation dictates fluid uptake and depends on the aldehyde:amine ratio. As this ratio is determined by the relative amounts of PEG and dextran, we examined the effect of PEG solid content on erosion over a range to the limit of PEG solubility (Fig. 2a). At solid content less than $10 \mathrm{wt} \%$, constructs lose structural integrity; at higher solid content, better stability is observed to a threshold value (above $20 \mathrm{wt} \%$ ). Each data set adhered to the expected erosion profile and fit a dual exponential decay model. In this model, polymer mass, $\mathrm{M}$, follows the erosion of two components - free $\left(\mathrm{M}_{1}\right)$ and crosslinked $\left(\mathrm{M}_{2}\right)$ material each defined by relative proportion of total mass, and a specific erosive rate constant, $\mathrm{k}$, at each phase $M=M_{1} \cdot e^{\left(-k_{1} t\right)}+M_{2} \cdot e^{\left(-k_{2} t\right)}$.

The relative measures of the mass components provide an indirect determination of gel content and degree of crosslinking. Though the ratio of unbound and bound rate constants $\left(\mathrm{k}_{1} / \mathrm{k}_{2}\right)$ spans an order of magnitude with changes in shape, in a fascinating manner the relationship between in vivo and in vitro ratios is virtually fixed at 2 (Table S1), providing a valuable means of assessing erosion in this system. The relative rates of erosion of crosslinked and free material might allow one to infer in vivo behavior from in vitro performance, and the very acceleration of in vitro erosion can be used to predict in vivo erosion. As the number of amine groups increases with PEG content, the unbound component drops exponentially (Fig. 2c) but overall erosion is still determined by the rate constant of the bound or crosslinked phase rate ( $\mathrm{k}_{2}$ ) (Fig. 2b). Erosion is limited by crosslinking and as the number of amine groups available for gel formation rises, erosion rate drops significantly. 
We validated this bi-exponential erosion model and model predictability by prospectively predicting adhesive erosion profiles for newly synthesized PEG:dextran formulations with 10 or 14\% PEG solid contents (formulations specified in Table S2). Erosive behavior was calculated using values for $\mathrm{M}_{1}, \mathrm{M}_{2}, \mathrm{k}_{1}$ and $\mathrm{k}_{2}$ extracted from the fits of data in figures $2 \mathrm{~b}-\mathrm{c}$ and correlated exceptionally with empiric measures of erosion (Fig. 2d, $\mathrm{R}=0.99$ ).

The highest benefit of such model would be the ability to predict in vivo erosion kinetics of newly synthesized materials from in vitro data. This will minimize the number of animals used and will also serve as a convenient screening platform in vitro. Interestingly, in vivo erosion (Figure 3a) followed the expected exponential decay and differed with PEG solid content material erosion as seen with disks in vitro (Figure 2a), establishing a fixed linear relationship between the in vitro and in vivo erosion versus time for all formulations (Figure $3 b$ ). The ratio of erosion rates in both domains correlates with PEG solid content (Figure $3 c$ ). This highly sensitive correlation was used to accurately predict in vivo performance based on in vitro data for all samples in this formulation type (Fig. 3d, R=0.99). Successful prediction of material erosion demonstrates continuity within the design space and the potential for strategic adjustment of PEG:dextran composition to meet specific requirements.

PEG:dextrans are dominated by hydrolytic erosive forces, other materials are subject to a more complex array of forces which often cannot be reproduced and whose impact is impossible to intuit. Fluorescent tracking enables us to assume that correlation will exist for specific environmental condition set and once attained, to inference that these are the physiologic values. We examined compressed collagen matrices, materials increasingly used in tissue engineering applications and whose degradation requires fluid imbibition, material swelling, enzyme penetration and enzymatic bulk degradation (Figure 4). As with hydrogels, the volume of diluent fluid is critical for erosion, but is increasingly complex in the case of enzymatic degradation. Diffusion of enzymes into samples and of degradation products out requires swelling. Hence, surrounding fluid volume determining degree of swelling should be considered in addition to the classic Michaelis-Menten enzyme kinetics ${ }^{18}$. Swelling of our collagen-based material is significantly affected by diluent volume with identical enzyme concentration (Supplementary Figure 4). While the subcutaneous space of a mouse has a limited fluid volume, the intraperitoneal cavity is significantly larger, with higher volume of fluids. Thus, not unexpectedly erosion kinetics in vitro correlated best with in vivo behavior for specific immersion volumes that varied with implant site (Figure 4). While in vitro erosion under $25 \mu \mathrm{l}$ diluent highly correlated with in vivo erosion in the subcutaneous space, linear relationship was obtained for intramuscular and intraperitoneal erosions when $100 \mu$ diluent volume was used in vitro. In a most intriguing manner the inferred volumes and concentration align remarkably with literature reported empirically-obtained values ${ }^{19}$.

As with the hydrogels, gravimetric analysis of collagen matrices underestimated erosion early and overestimated erosion late. Sample drying shifted these measures to coincide with fluorescent assays once again verifying the contamination of signal with swelling. The gravimetric analysis, however, was further limited as increasingly sensitive scales would need to be used to track sample weight changes in the regime where fluorescent quantification remains constantly sensitive (Supplementary Figure 5). 
Tracking material erosion is challenging. In vivo performance rarely coincides with erosion in vitro. Material fate varies with material dimensions, crosslinking, and composition, and environmental conditions and stresses in vitro that do not necessarily represent the in vivo state. Pathophysiologic conditions like inflammation cannot be recapitulated in vitro. Two questions arise-will erosion in vivo follow erosion in vitro even if they occur at different rates? And, if there is a match between performances in the two domains, can one be predicted from the other? Indeed, one could use in vitro behavior as a surrogate for erosion in vivo if such a correlation exits. Here we report that intravital tracking of fluorescent tags on degradable materials can be used similarly in vivo and in vitro, with a minimum of samples, sequentially on the same specimen, with significant accuracy and most importantly in a correlative fashion. In vivo erosion of our selected materials that degrade primarily by hydrolytic or enzymatic degradation correlates with in vitro erosion, allowing prediction of the former from the latter. Moreover, erosive materials can be considered like scaffoldtethered drugs ${ }^{20}$, where erosion kinetics like release kinetics can be successfully modeled with a dual exponent system with free and crosslinked polymer elements like free and bound drug (Fig. 1). Such a system can also now explain the impact of material shape and dimensions, and material formulation protocols on erosion profiles (Figs. 2-3) facilitating the design of materials with programmed retention time. Equally important is that such a system adds great insight into the mechanism of erosion and the physiological conditions that need to be established in vitro to attain correlation. We can now explain how and why materials implanted in different target sites, including the subcutaneous, intramuscular and intraperitoneal spaces, resulted in distinctive erosion profiles. While enzymatic degradation of materials in vivo is very complex, under the specific set of conditions we have been using, collagen-based material degradation in vivo could be recapitulated in vitro via variation in fluid volume with physiological enzyme concentrations (Figure 4a). It is remarkable how the definition of the in vivo conditions leads to linear correlation between in vivo and in vitro erosion profiles (Figure $4 b-c)$.

The ability to detect and predict the time course of in vivo erosion is crucial to the design and informed regulation and use of increasing number of biomedical devices with erosive properties. While the data published in this manuscript are material and conditions-specific, our approach can be adapted to track the erosion of multiple material systems. Moreover, erosion tracking can be extended beyond the hydrolytic and enzymatic processes examined here to include complex conjugations. Use of multiple tags can enable examination of material dynamics, and even the interaction between molecules using advanced fluorescent methods, such as Fluorescent Resonance Emission Transfer (FRET). The incorporation of multiple concomitant tags allows independent tracking and correlation of drug release and material loss from a polymer drug-eluting scaffold, ${ }^{21-23}$ or the fate of cells and materials within tissue engineered formulations. ${ }^{24,25}$ When tagging is not possible, one could entrap fluorescent molecules e.g., quantum dots, that would serve as surrogate for erosion. It is now possible to follow the fate of erosive materials in a precise and reproducible manner that can be used in vivo and in vitro and is amenable to mathematical modeling and prospective prediction. 


\section{Methods}

\section{Materials' selection and characterization are described in the Supplementary Information}

\section{Quantifying material erosion using fluorescent signal ex-vivo-Mass loss}

kinetics as measured by gravimetric analysis was compared with erosion profiles obtained by following fluorescent signal loss of labeled materials. To enable erosion detection via optical imaging, PEG was labeled with fluorescein and collagen with Texas Red.

Fluorescein labeled PEG was synthesized as follows: 2.4g PEG amine was dissolved in 6 $\mathrm{mL}$ dichloromethane. Fluorescein-5-carboxyamido hexanoic acid (Invitrogen) was added to the solution followed by addition of $12 \mu \mathrm{L}$ triethylamine (Sigma). The mixture was stirred at room temperature for 48 hours. The solid that remained after solvent evaporation was dissolved in $100 \mathrm{~mL}$ dd water, dialyzed and lyophilized. PEG solution containing $1 \%$ fluorescent PEG was mixed with dextran aldehyde to prepare fluorescent gels. While materials were submerged in $2 \mathrm{ml} \mathrm{PBS}$ and shaken at $37^{\circ} \mathrm{C}$, media was exchanged daily and fluorescence intensity in the media was measured at an excitation wavelength of $485 \mathrm{~nm}$ and emission wavelength of $538 \mathrm{~nm}$. The erosion profile was followed by quantifying the loss of fluorescent signal with time.

Texas Red® labeled collagen was synthesized by reacting the collagen sponges with Texas Red@-T succinimidyl ester ( $5.6 \mu \mathrm{l}$ per sponge $)$ in $0.2 \mathrm{M}$ bicarbonate buffer $(0.320 \mathrm{ml}$ per sponge), for 2 hours at room temperature. The sponges were rinsed three times in PBS to remove excess unreactive dye.

Stability of PEG-fluorescein bond-To verify that the fluorescent tag remains attached to the polymer materials $1 \mathrm{ml}$ PEG-F* was placed in a dialysis membrane of cellulose ester (Spectra/Por Biotech, MWCO=1000, \#131096) surrounded by 100ml PBS 1x. Free fluorescein could then diffuse out and the media was scanned intermittently at excitation and emission of $485 / 538 \mathrm{~nm}$ to quantify the amount of free or detached tag with time.

Quantifying material erosion using fluorescent signal in-vivo-Sterile solutions passed through $0.2 \mathrm{~mm}$ filters were used to prepare PEG:dextran constructs in the shapes of disks, blocks and coated mesh cylinders of the same dimensions as used for in vitro evaluation. The constructs were implanted subcutaneously into isoflurane-anesthetized nude albino mice (Charles River Labs) through a $1 \mathrm{~cm}$ skin incision into the dorsal subcutaneous space of the mice. The incision and pocket were closed with 7-0 prolene sutures.

Sterile collagen based samples, $4 \mathrm{~mm}$ in diameter were similarly implanted into the subcutaneous space of a mouse (SC), intraperitoneally (IP) and intramuscularly (IM). Immediately and at various intervals after surgery, the animals were imaged using the Xenogen IVIS device using the relevant filter sets. Fluorescence intensity was determined by calculating the efficiency overlying each construct where the fluorescent intensity is corrected to eliminate tissue autofluorescence. While fluorescein was used for SC implantations, Texas Red was used for deeper target sites (IM and IP) to avoid photo bleaching and light penetration issues. The choice of fluorescent dye is critical, especially when tracking materials for extended period of times. Optimal dyes are near-IR dyes that do 
not undergo photobleaching during the experimental period and do not overlap with tissue autofluorescence.

Statistical Analyses-Data are presented as mean \pm standard deviation unless otherwise indicated ( $\mathrm{n}=3$ for in vitro swelling and degradation, adhesion and gelation time of PEGdextran formulations, $\mathrm{n}=5$ for in vitro erosion characterization of collagen based samples, $\mathrm{n}=5$ and 10 for in vivo erosion tracking of PEG:dextran and collagen based formulations, respectively). To take multiple comparisons into account, when comparing swelling and degradation, statistical comparisons were done using two way ANOVA with replication. A $\mathrm{P}$-value $<0.05$ was considered to denote statistical significance.

\section{Supplementary Material}

Refer to Web version on PubMed Central for supplementary material.

\section{Acknowledgments}

The authors acknowledge the NIH (GM/HL 49039, UL1 RR 025758 to ERE) and the MIT Deshpande center for the financial support of this work. We would like to thank Dr. Jeffrey Karp, Dr. Erel Levine and Maria Carcole for assisting in the preparation of the manuscript.

\section{References}

1. Bruggeman JP, de Bruin BJ, Bettinger CJ, Langer R. Biodegradable poly(polyol sebacate) polymers. Biomaterials. 2008; 29:4726-4735. [PubMed: 18824260]

2. Grayson AC, et al. Differential degradation rates in vivo and in vitro of biocompatible poly(lactic acid) and poly(glycolic acid) homo- and co-polymers for a polymeric drug-delivery microchip. Journal of biomaterials science. 2004; 15:1281-1304.

3. Benny O, et al. In vivo fate and therapeutic efficacy of PF-4/CTF microspheres in an orthotopic human glioblastoma model. Faseb J. 2008; 22:488-499. [PubMed: 17873103]

4. Gopferich A. Mechanisms of polymer degradation and erosion. Biomaterials. 1996; 17:103-114. [PubMed: 8624387]

5. Anseth KS, Shastri VR, Langer R. Photopolymerizable degradable polyanhydrides with osteocompatibility. Nature biotechnology. 1999; 17:156-159.

6. Falco EE, Patel M, Fisher JP. Recent Developments in Cyclic Acetal Biomaterials for Tissue Engineering Applications. Pharmaceutical research. 2008

7. Lee H, et al. Local delivery of basic fibroblast growth factor increases both angiogenesis and engraftment of hepatocytes in tissue-engineered polymer devices. Transplantation. 2002; 73:15891593. [PubMed: 12042644]

8. Little SR, et al. Poly-beta amino ester-containing microparticles enhance the activity of nonviral genetic vaccines. Proceedings of the National Academy of Sciences of the United States of America. 2004; 101:9534-9539. [PubMed: 15210954]

9. Ifkovits JL, Burdick JA. Review: photopolymerizable and degradable biomaterials for tissue engineering applications. Tissue engineering. 2007; 13:2369-2385. [PubMed: 17658993]

10. Mahdavi A, et al. A biodegradable and biocompatible gecko-inspired tissue adhesive. Proceedings of the National Academy of Sciences of the United States of America. 2008; 105:2307-2312. [PubMed: 18287082]

11. Bettinger CJ, Bruggeman JP, Borenstein JT, Langer RS. Amino alcohol-based degradable poly(ester amide) elastomers. Biomaterials. 2008; 29:2315-2325. [PubMed: 18295329]

12. Witt C, Mader K, Kissel T. The degradation, swelling and erosion properties of biodegradable implants prepared by extrusion or compression moulding of poly(lactide-co-glycolide) and ABA triblock copolymers. Biomaterials. 2000; 21:931-938. [PubMed: 10735470] 
13. Kim J, et al. Synthesis and evaluation of novel biodegradable hydrogels based on poly(ethylene glycol) and sebacic acid as tissue engineering scaffolds. Biomacromolecules. 2008; 9:149-157. [PubMed: 18072747]

14. Chao GT, et al. Synthesis, characterization, and hydrolytic degradation behavior of a novel biodegradable $\mathrm{pH}$-sensitive hydrogel based on polycaprolactone, methacrylic acid, and poly(ethylene glycol). Journal of biomedical materials research. 2008; 85:36-46. [PubMed: 17688254]

15. Kost J, Leong K, Langer R. Ultrasound-enhanced polymer degradation and release of incorporated substances. Proceedings of the National Academy of Sciences of the United States of America. 1989; 86:7663-7666. [PubMed: 2813349]

16. Artzi N, et al. Characterization of Star Adhesive Sealants Based On PEG/Dextran Hydrogels. Macromolecular bioscience. 2009

17. Artzi N, Shazly T, Baker AB, Bon A, Edelman ER. Aldehyde-amine chemistry enables modulated biosealants with tissue-specific adhesion. Adv Mater. 200910.1002/adma.200900340

18. Tzafriri AR, Bercovier M, Parnas H. Reaction diffusion model of the enzymatic erosion of insoluble fibrillar matrices. Biophysical journal. 2002; 83:776-793. [PubMed: 12124264]

19. Hartveit F, Thunold S. Peritoneal fluid volume and the oestrus cycle in mice. Nature. 1966; 210:1123-1125. [PubMed: 6007179]

20. Tzafriri AR. Mathematical modeling of diffusion-mediated release from bulk degrading matrices. J Control Release. 2000; 63:69-79. [PubMed: 10640581]

21. Shi X, et al. Novel mesoporous silica-based antibiotic releasing scaffold for bone repair. Acta biomaterialia. 2009; 5:1697-1707. [PubMed: 19217361]

22. Zhang C, et al. Loading dependent swelling and release properties of novel biodegradable, elastic and environmental stimuli-sensitive polyurethanes. J Control Release. 2008; 131:128-136. [PubMed: 18703098]

23. Nuttelman CR, Tripodi MC, Anseth KS. Dexamethasone-functionalized gels induce osteogenic differentiation of encapsulated hMSCs. Journal of biomedical materials research. 2006; 76:183195. [PubMed: 16265650]

24. Riboldi SA, Sampaolesi M, Neuenschwander P, Cossu G, Mantero S. Electrospun degradable polyesterurethane membranes: potential scaffolds for skeletal muscle tissue engineering. Biomaterials. 2005; 26:4606-4615. [PubMed: 15722130]

25. Levenberg S, et al. Engineering vascularized skeletal muscle tissue. Nature biotechnology. 2005; 23:879-884. 

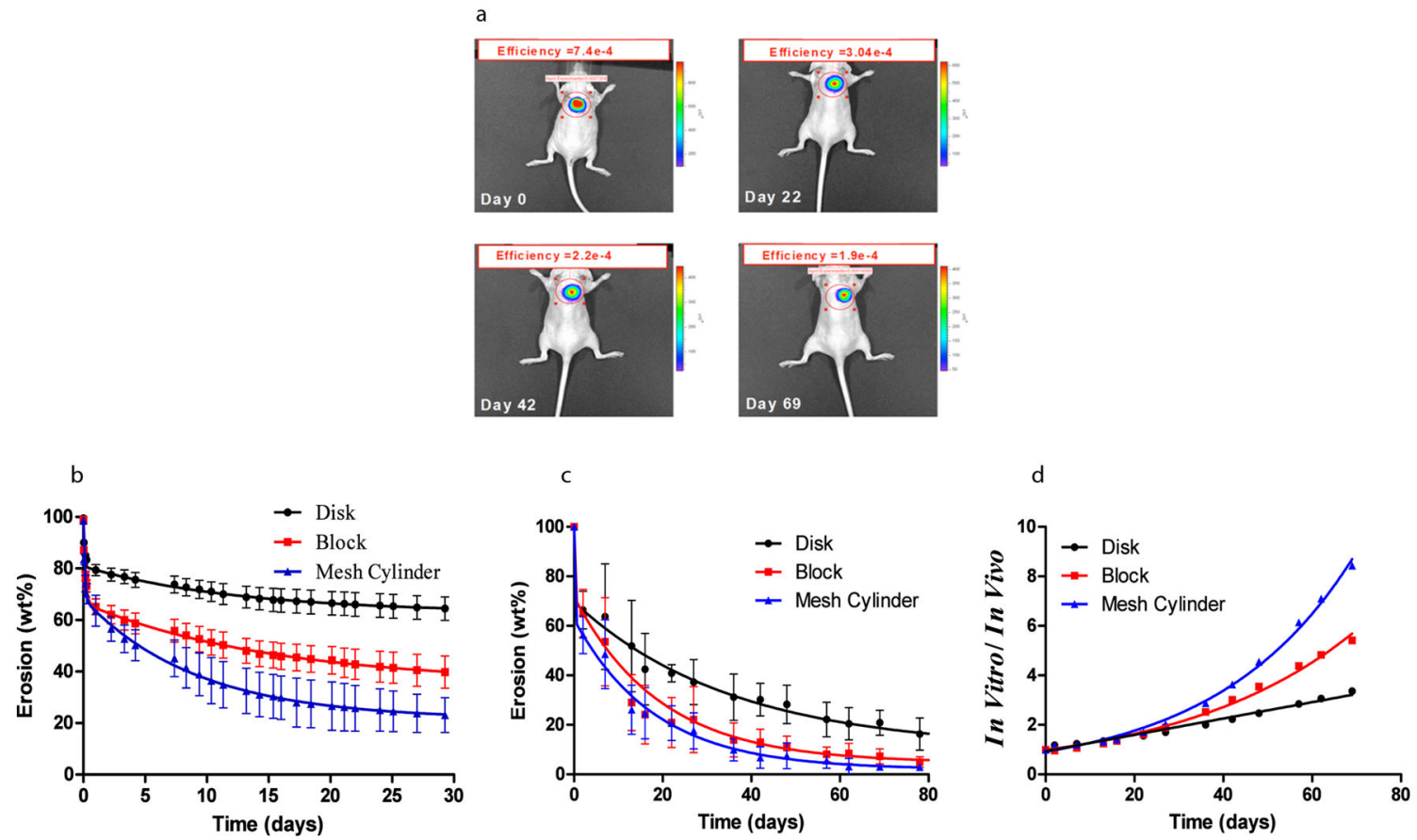

Figure 1. In vitro - in vivo correlation of PEG:dextran erosion profiles exists and varies with material surface area

In vitro and in vivo erosion profiles of PEG-dextran (D10-50-14 P8-10-40) cast in a series of shapes are depicted by tracking the loss of fluorescence intensity with time. The effect of material shape on degradation profile was followed in vivo noninvasively in the dorsal subcutaneous space of the mouse (disk shaped materials are presented) (a), and in vitro (b). The loss of fluorescent signal with time in vivo was converted to weight loss (c) and correlated with the in vitro loss (d). An excellent correlation was found between mean values of in vitro and in vivo erosions of disk $\frac{\text { In }- \text { vitro }}{\text { In }- \text { vivo disk }}=0.033 t+0.93 ;(\mathrm{R}=0.96)$, block $\frac{\text { In }- \text { vitro }}{\text { In }- \text { vivo block }}=0.928 \cdot \exp (0.27 \cdot t) ;(\mathrm{R}=0.94)$, and mesh cylinder $\frac{\text { In - vitro }}{\text { In - vivo } \text { mesh }}=0.934 \cdot \exp (0.032 \cdot t) ;(\mathrm{R}=0.98)$. Disk erosion in vivo tracked linearly with erosion in vitro (Figure 1d, $\mathrm{R}=0.98$ ). Thin blocks and mesh cylinders with significantly higher surface area than the disks $\left(116,190\right.$ and $86 \mathrm{~mm}^{2}$ respectively) showed accelerated erosion in vitro compared with the erosion in vivo, leading to exponential dependence of the ratio between in vivo and in vitro erosion with time (Figure $1 \mathrm{~d}, \mathrm{R}=0.99$ for both shapes). 

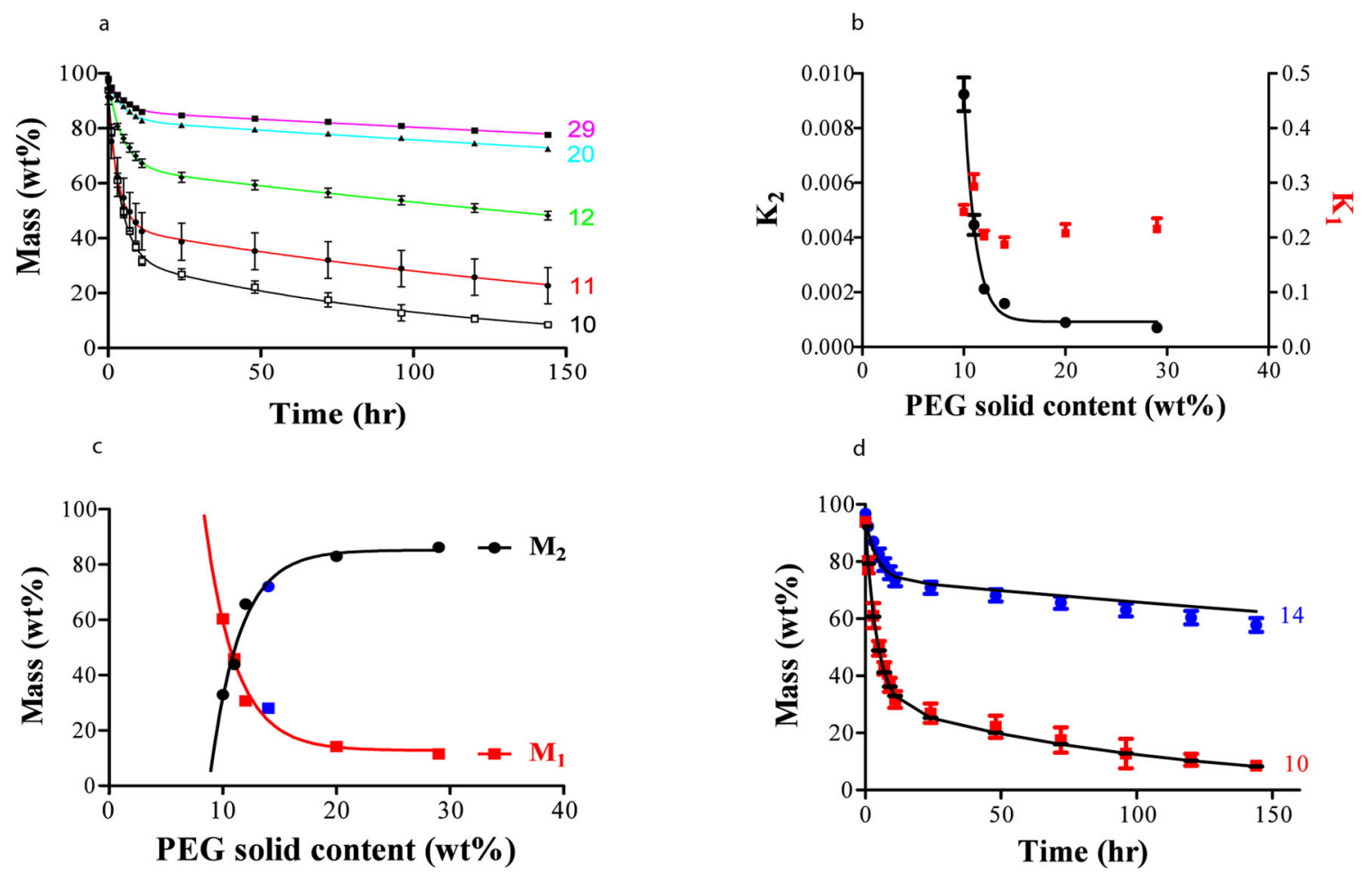

Figure 2.

Dual exponential decay model describes PEG:dextran in vitro erosion and enables prospective prediction of erosion profile of new material formulations. Alteration of PEG solid content from 10 to $29 \mathrm{wt} \%$ enables fine-tuning of material erosion kinetics (a). Model descriptors are presented as a function of PEG solid content. While $\mathrm{k}_{1}$ is constant and $\mathrm{k}_{2}$ decays exponentially (b); $\mathrm{M}_{1}$ and $\mathrm{M}_{2}$ similarly demonstrate reciprocal exponential changes with PEG solid content (c). The relationships between model descriptors and PEG solid content $\left(\mathrm{PEG}_{\mathrm{SC}}\right)$ are as follows:

$$
\begin{gathered}
k_{2}=53.7 \cdot \exp \left(-0.88 \cdot\left(P E G_{S C}\right)\right)+0.0009, \\
M_{1}=3979 \cdot \exp \left(-0.44 \cdot\left(P E G_{S C}\right)\right)+12.4 \text { and } \\
M_{2}=-3028 \cdot\left(1-\exp \left(-0.41 \cdot\left(P E G_{S C}\right)\right)+85.4 .\right.
\end{gathered}
$$

Using the equations describing the relation between model descriptors and PEG solid content, the erosion profile of new compositions containing 10 and 14wt\% PEG were prospectively predicted (points are empirically accumulated and lines are model predictions) (d). Constants were extrapolated from the data fits and are inserted in the figures as blue symbols, while squares represent $\mathrm{k}_{1}$ and $\mathrm{M}_{1}$ and circles represent $\mathrm{k}_{2}$ and $\mathrm{M}_{2}$. Predicted erosion correlated well with empiric observations (Pearson's coefficient $\mathrm{R}=0.99$ ). 

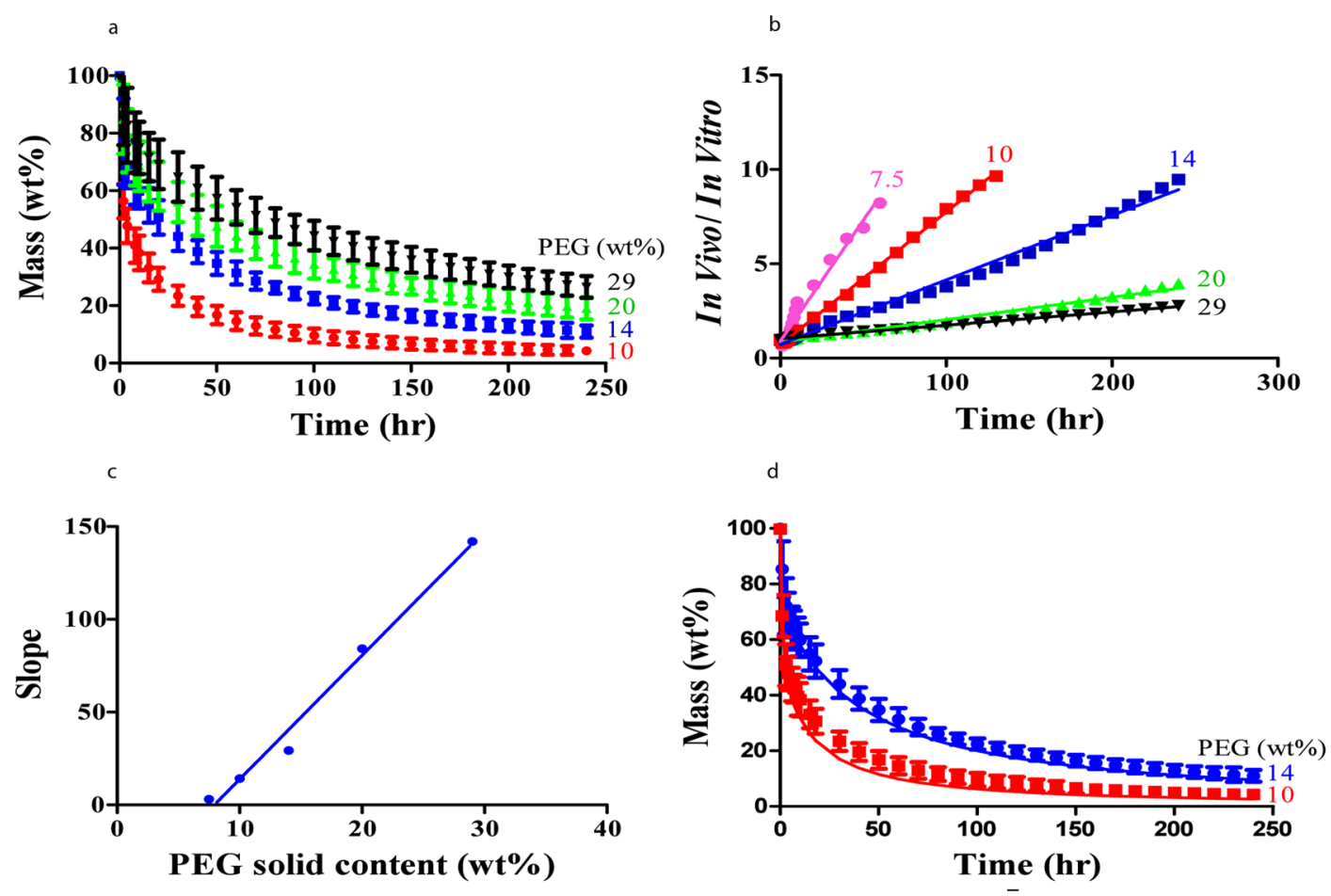

Figure 3. In vitro- in vivo correlation of PEG:dextran erosion profiles enables prediction of in vivo erosion kinetics from in vitro data

In vivo erosion of PEG-dextran formulations is depicted and follows the trend of the in vitro erosion profile, at a faster pace, as demonstrated for compositions with 10, 14, 20 or $29 \mathrm{wt} \%$ PEG solid content (a). A linear relationship exists between the ratio of in vivo and in vitro erosion as a function of time for all PEG solid contents examined (b). The slopes of these curves linearly correlate with PEG solid content (c). Using this linear relationship, in vivo erosion profiles of two different formulations were accurately predicted from in vitro data $(\mathrm{R}=0.99)$ (points are empirically accumulated and lines are model predictions) $(\mathrm{d})$. 


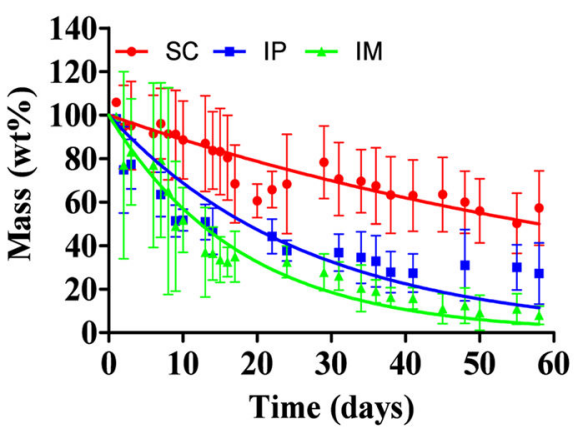

$b$

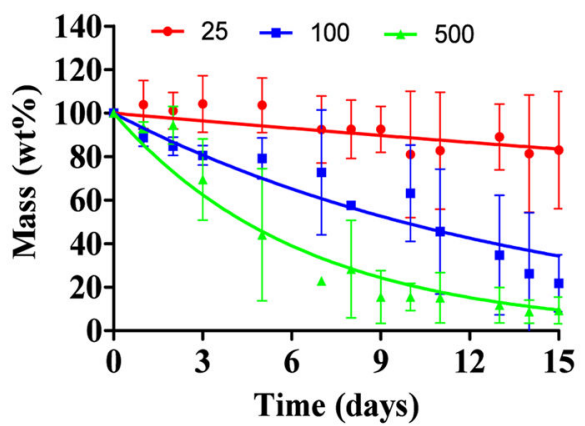

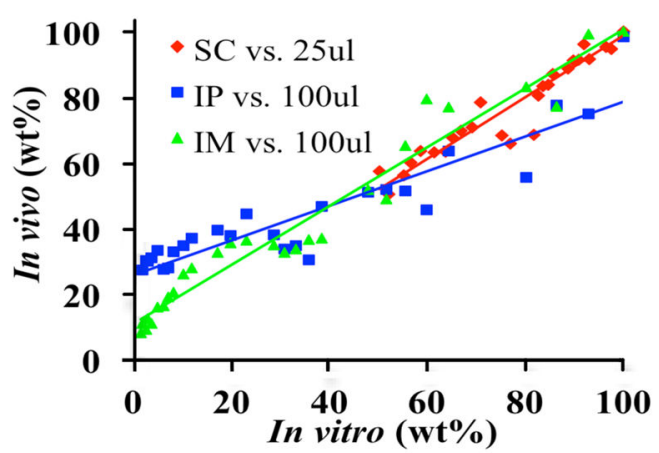

Figure 4. In vivo erosion profile is site-dependant and can be used to infer physiologically relevant in vitro conditions for enzymatic materials

In vitro and in vivo erosion of compressed denatured type II collagen are presented. In vivo erosion at target sites that differ in enzyme concentration and fluid volume (Subcutaneous (SC), intraperitoneal (IP) and intramuscular (IM)) is site dependent and fits one exponential decay model ( $R=0.44,0.77$, and 0.7 for $S C, I P$ and $I M)$ (a). The in vivo erosion profiles were used to infer physiologically relevant conditions of diluent volume and enzyme concentration. While in vitro erosion depends both on enzyme concentration and fluid volume, a specific set of conditions resulted in an in vitro erosion profile $(R=0.71,0.9$, and 0.95 for $S C, I P$ and IM) (b) that linearly correlates with the in vivo erosion (c). The erosion in both domains fit an exponential decay model. A correlation between the erosion profiles in vitro and in vivo is achieved when SC implantation is plotted versus in vitro erosion of material submerged in $25 \mu \mathrm{l}$ of PBS solution containing physiological concentration of collagenase, and for IM and IP implantations when compared with in vitro erosion using $100 \mu \mathrm{l}$ of PBS containing enzyme solution. Linear correlation between in vitro and in vivo erosion enables screening of materials in vitro with in vivo prediction capacity. 